\title{
Comparison and analysis of the compression algorithm based on DCT and DWT warehousing image
}

\author{
Guo Jian ${ }^{1,}$ a , Lv Xianwei ${ }^{2, \text { b }}$ \\ ${ }^{1}$ Office of teaching affairs, Beijing Wuzi University, Beijing, 101149, \\ China \\ ${ }^{2}$ Graduate faculty, Beijing Wuzi University, Beijing, 101149, China \\ aemail: guojian760118@163.com, ${ }^{b}$ email:419298656@qq.com
}

\begin{abstract}
In order to ensure safe operation of the storage system, Warehousing environment for real-time monitoring of effective security is very important. Due to the huge amount of image data warehouse monitoring system acquisition, image acquisition of the warehouse for effective compression become hot. This paper describes the discrete cosine transform (DCT) and discrete wavelet transform (DWT) of the realization of the principle and its implementation process, And the use of technology tools based on these algorithms for storage image compression and reduction, Then compared and analyzed based on the experimental results, To further enhance storage safety monitoring system provides the foundation.

Keywords: Warehousing image; discrete cosine transform; discrete wavelet transform; image compression

\section{Introduction}

With the international market and the continuous development of China's logistics industry, Warehousing has become an important part of the logistics process. In the warehouse control system, due to the huge collection of image data is difficult to transport and store, which greatly restricted the development of warehouse control system, So the effective image compression is very important in the warehouse monitoring.

The purpose of image compression is to run out of a larger image of the original small amount of bytes and transmission, and requires a better quality of the restored image.

In this paper, aiming at the hotspot of image compression, discrete cosine transform (Discrete Cosine Transform, DCT) and discrete wavelet transform (Discrete Wavelet Transform, DWT) comparison and analysis of two compression for storage image.
\end{abstract}




\section{Image compression based on DCT and DWT}

\subsection{Discrete cosine transform}

DCT (Discrete Cosine Transform, discrete cosine transform) is proposed by Ahmed and Rao in the twentieth Century 70's , It has proved to be a sub-optimal orthogonal transformation under conditions of minimum mean square error. DCT can use to convert an image from the spatial domain into the frequency domain transform, the energy of the image is relatively concentrated distribution in the frequency domain, laying the foundation for future compression. DCT is used widely at this stage image compression technology, has become the core of many international image coding standard [1]. JPEG (Joint Photographic Experts Group, Joint Photographic Experts Group) [2] is the most commonly used lossy image compression format, images can be compressed in a small storage space, and ensure the quality of the image. Standard JPEG encoder is based on the DCT, and was compressed by quantization and entropy coding in the transformation based on. Gray image is shown in Figure 1 is a block diagram of codec system of JPEG image compression based on DCT.

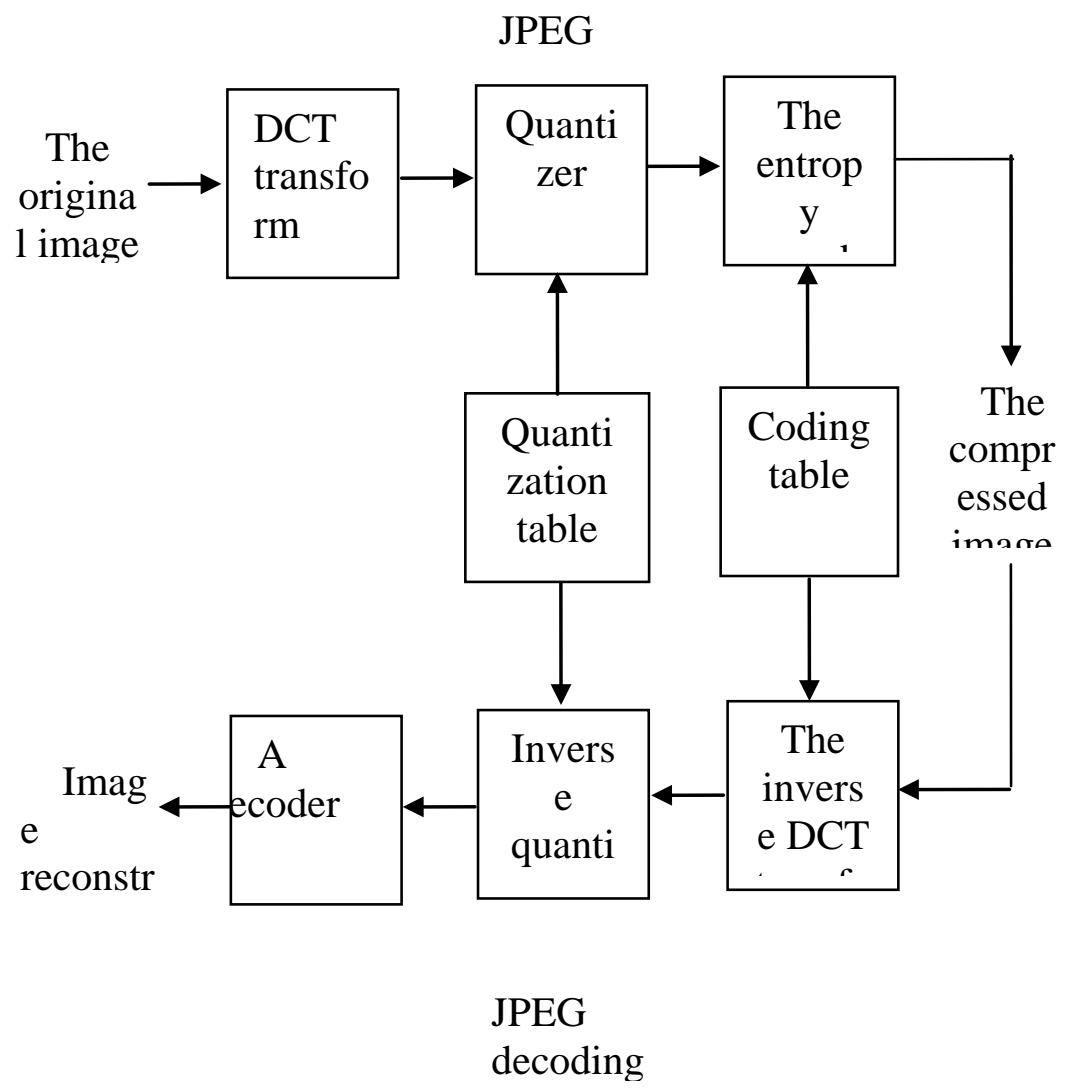

Figure 1. A block diagram of codec system of JPEG image compression based on 
In the JPEG compression algorithm, DCT the original image is first divided into $\mathrm{N} \times \mathrm{N}(\mathrm{N}=4,8,16)$ sub-blocks, each sub-block are separately transformed. $\mathrm{N} \times \mathrm{N}$ sub-blocks of two-dimensional DCT is defined as follows:

Two-dimensional discrete cosine transformation formula:

$$
F(u, v)=c(u) c(v) \frac{2}{N} \sum_{x=0}^{N-1} \sum_{y=0}^{N-1} f(x, y) \cos \left(\frac{2 x+1}{2 N}\right) \cos \left(\frac{2 y+1}{2 N} v \pi\right)
$$

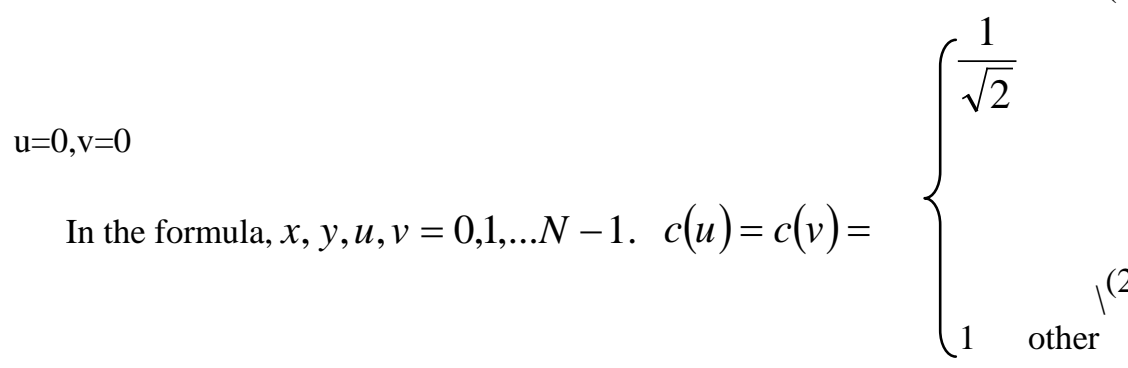

Other two-dimensional inverse discrete cosine transform formula is:

$f(x, y)=\frac{2}{N} \sum_{u=0}^{N-1} \sum_{v=0}^{N-1} c(u) c(v) F(u, v) \cos \left(\frac{2 x+1}{2 N} u \pi\right) \cos \left(\frac{2 y+1}{2 N} v \pi\right)$

In the formula, $x, y, u, v=0,1, \ldots N-1 . \quad c(u)=c(v)=\left\{\begin{array}{cc}\frac{1}{\sqrt{2}} \mathrm{u}=0, \mathrm{v}=0 \\ 1 & \text { other }\end{array}\right.$

Then generates $\mathrm{N}^{*} \mathrm{~N}$ DCT coefficient, one of the first for the DC coefficients, the rest $\mathrm{N}^{*} \mathrm{~N}-1$ for AC coefficient, according to the quantization table to quantify the DCT coefficients, the final will be quantization DCT coefficient of entropy coding are compressed data. Similarly, the JPEG image decoding process, the data is sequentially compressed entropy decoding, inverse quantization, inverse discrete cosine transform algorithm for the DCT coefficients into blocks of $\mathrm{N} * \mathrm{~N}$ image, the final reconstructed approximation of the original image.

\subsection{Discrete Wavelet Transform}

Wavelet analysis technology and multi-resolution analysis theory developed in recent years, becomes a powerful tool for research on image compression. 
Image compression based on the discrete wavelet transform has many new features, such as high compression ratio, also supports lossless and lossy compression, progressive transmission, ROI ,etc[4]. The basic idea of wavelet transform is that the signal is expanded into a weighted sum of a family of base functions, using a function to express the signal function or approximation, this family of functions is obtained by the basic function of the translation and dilation.

JPEG 2000 is the Joint Photographic Experts Group by ISO and CCITT / ITU co-founded (JPEG), launched at the end of 2000 as a still image compression standard based on wavelet transform (ISO / IEC 15444-1 12, ITU T.800 808).

In the core of the JPEG 2000 codec system, the lossy compression used is based on lifting Daubechies 9/7 Filters achieved DWT, lossless compression is used to enhance Le Gall 5/3 Filters achieved based the DWT.

FDWT (forward discrete wavelet transform) of the conversion formula is: ( $\mathrm{j}$ $=0 \sim \mathrm{NL})$

$$
\begin{aligned}
& W_{2^{I}} x(n)=\sum_{k \in Z} h_{H}(k) \times S_{2^{I-1}} x\left(n-2^{j-1} k\right) \\
& S_{2^{I}} x(n)=\sum_{k \in Z} h_{L}(k) \times S_{2^{I-1}} x\left(n-2^{j-1} k\right)
\end{aligned}
$$

Among them, $\mathrm{X}$ is the original data, $\mathrm{D}=\mathrm{Wx}$ for difference - the high frequency part, $\mathrm{A}=\mathrm{Sx}$ for residual - the low frequency part, $\mathrm{hL}$ and $\mathrm{hH}$ for the decomposition of a low-pass filter and high pass filter coefficient . See figure 2 / 3.

\begin{tabular}{l} 
0L (raw data $x$, including all kinds of \\
frequency information) \\
\hline 1L (At the end of the low frequency \\
\hline 2L (single frequency) \\
\hline 3L (lowest frequency)
\end{tabular}

$\mathrm{L}=$ Low frequence, $\mathrm{H}=$ High frequence

Figure 2. Wavelet decomposition process step by step (NL = 3)

\begin{tabular}{|l|l|l|lc|}
\hline \multicolumn{4}{|c|}{ X } \\
\hline \multicolumn{3}{|c|}{$\downarrow$ FDWT } \\
\hline $3 \mathrm{~L}$ & $3 \mathrm{H}$ & $2 \mathrm{H}$ & $1 \mathrm{H}$ \\
\hline
\end{tabular}

Figure 3. Level 3 wavelet decomposition 
Visible, every time (such as the $n+1$ ) wavelet transform will make the original low frequency subband blocks (n LL) to produce three high frequency subband blocks:: $(n+1) \mathrm{LH},(\mathrm{n}+1) \mathrm{HL}$ and $(n+1) \mathrm{HH}$, so the total number of NL transform the subband block is $3 \times \mathrm{NL}+1$. For example, get 2 transform $(3 \times$ $2+1=7$ ) 7 sub block (see Figure 4), get 3 transform $(3 \times 3+1=10) 10$ sub block.

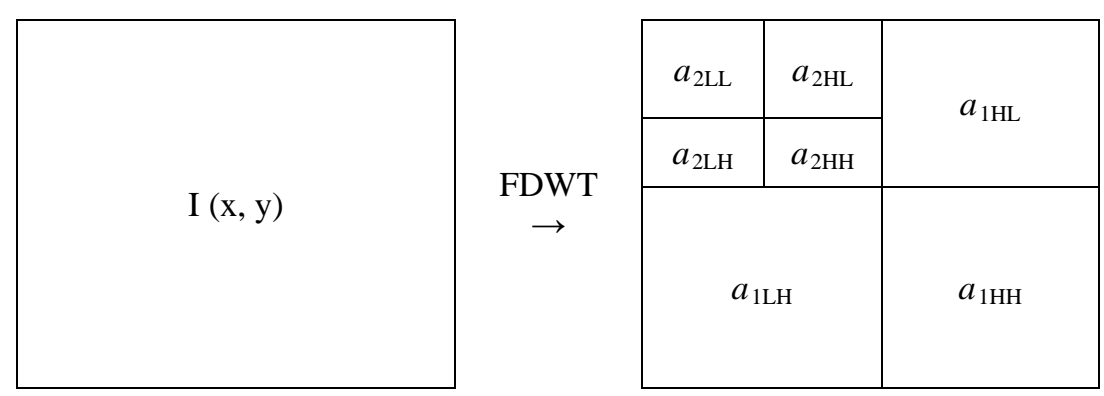

Figure 4. Schematic diagram of 2D FDWT process $(\mathrm{NL}=2)$

Transform formula of inverse transformation IDWT (reconstruction) is (using positive transformation has preserved all the $S_{2^{J}} X$ and $W_{2^{j}} X:(\mathrm{j}=\mathrm{NL}$ $\sim 0)$

$$
\begin{aligned}
& S_{2}^{I-1} x(n)=\sum_{k \in Z} g_{L}(k) \times S_{2^{i}} x\left(n-2^{j-1} k\right)+ \\
& \sum_{k \in Z} g_{H}(k) \times W_{2^{i}} x\left(n-2^{j-1} k\right) \\
& x(n)=S_{2} x(n)
\end{aligned}
$$

Among them, the gL and gH for synthesis using low-pass filter $\overline{H(w)}$ and high-pass filter $\overline{G(W)}$ coefficient of symmetry position. See figure 5/6

Image reconstruction requires IDWT (Inverse Discrete Wavelet Transformation inverse discrete wavelet transform) process, it is just the inverted FDWT process. In the JPEG 2000, however, does not directly use filter coefficient for DWT, but using the filter improve implementation for DWT. 


\begin{tabular}{|l|}
\hline $\begin{array}{l}\text { OL (raw data } \mathrm{x} \text {, including all kinds of } \\
\text { frequency information) }\end{array}$ \\
\hline 1L (At the end of the low frequency) \\
\hline 2L (single frequency) \\
\hline 3L (lowest frequency) \\
$\begin{array}{c}\text { 3H ( At the end of the high } \\
\text { frequency) }\end{array}$ \\
\hline
\end{tabular}

$\mathrm{L}=$ Low frequence, $\mathrm{H}=$ High frequence

Figure 5. wavelet reconstruction process step by step $(\mathrm{NL}=3)$

\begin{tabular}{|c|c|c|c|}
\hline \multicolumn{4}{|c|}{$X$} \\
\hline \multicolumn{4}{|c|}{ 个FDWT } \\
\hline 3L & $3 \mathrm{H}$ & $2 \mathrm{H}$ & $1 \mathrm{H}$ \\
\hline
\end{tabular}

Figure 6. level wavelet reconstruction

\section{MATLAB simulation experiment}

This paper selects a pair of $168 \times 166$ storage goods images as the experimental object, the simulation experiments are carried out with MATLAB 7.11, using the DCT and the DWT two kind of transformation of the original image.

\section{1 discrete cosine transform}

Figure 7 is formed by the compression storage image results using different quantization table, through the comparison of different compression ratio is very easy to the quality of image compression.

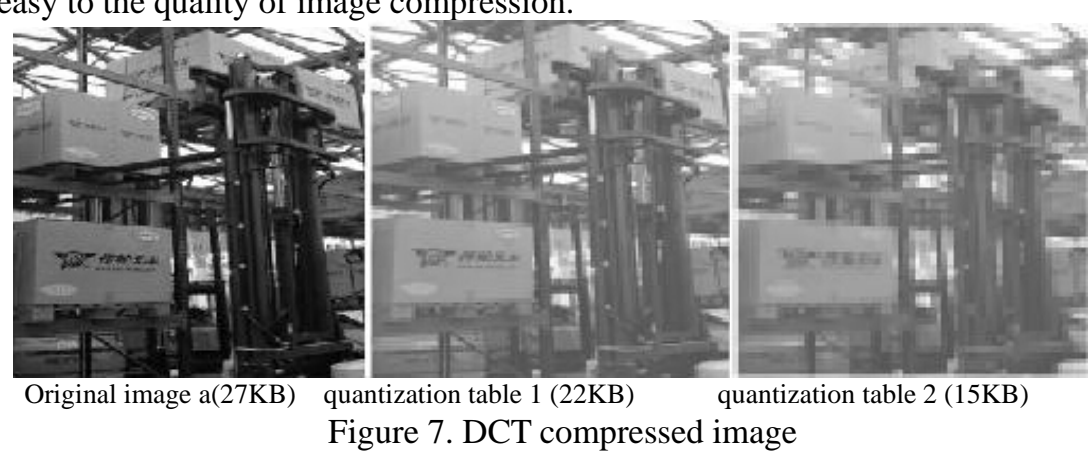


Image compression results show that when the image compression ratio increases, the image distortion is serious, easy to produce block effects.

\subsection{Discrete wavelet transform image}

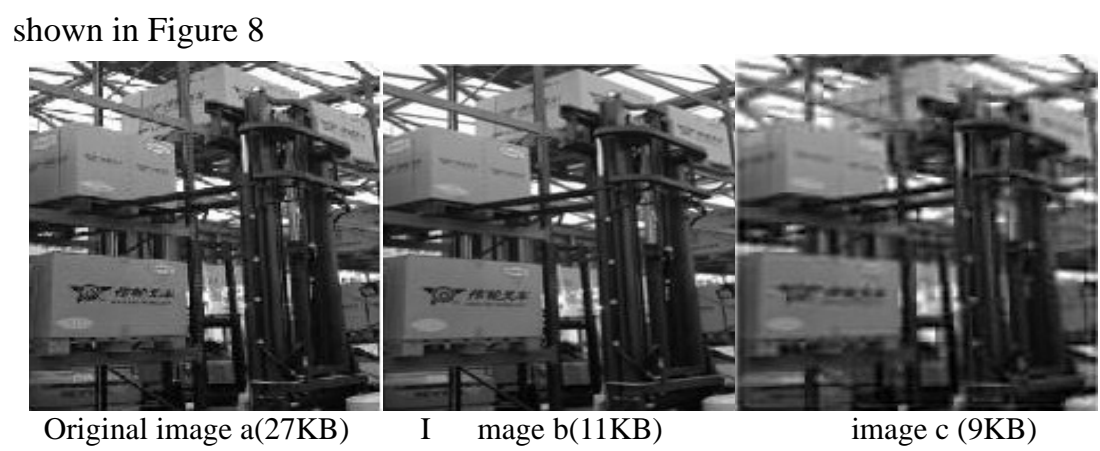

Figure 8. DWT image compression

In this paper, by using the software of MATLAB three level wavelet image decomposition of storage, the three level wavelet decomposition procedure is similar, so only list storage image level wavelet decomposition process, the compression effect as shown in Figure 8 (b)

$\mathrm{X}=$ imread('F: 'cangchu.jpg');

\%Read storage image

$\mathrm{X}=\operatorname{rgb} 2 \operatorname{gray}(\mathrm{X})$

subplot(221);imshow(X);

title('The original storage image');

[C,S]=wavedec2(X,3,'db1'); wavelet decomposition

ca1 = appcoef2(C,S,'db1',1);

extracted in scale 1

ch1 = detcoef2('h',C,S,1);

coefficient of horizontal scale 1

$\mathrm{cv1}=\operatorname{detcoef} 2\left({ }^{\prime} \mathrm{V}, \mathrm{C}, \mathrm{S}, 1\right)$;

coefficient of vertical scale 1

cd1 = detcoef2('d',C,S,1);

high frequency coefficient

$\mathrm{A} 1=[\mathrm{ca} 1, \mathrm{ch} 1 ; \mathrm{cv} 1, \mathrm{~cd} 1]$;

subplot(222),imshow(A1,[ ] ); $\quad$ \%Show storage image a wavelet decomposition

title('Storage image one level wavelet decomposition')

Image compression results show that wavelet transform is better for the storage effect of image compression, image compression effect to meet the requirements of storage security surveillance for clarity.

\subsection{Comparison and analysis of DCT and DWT}

DWT similar effect on image compression in the DCT, are trying to before 
quantization and entropy code as much as possible to reduce the spatial redundancy information of pixels, so as to improve the image compression ratio. However, there are big differences in specific methods, this mainly displays in two aspects as follows:

(1)The basic function of DCT is the cosine function periodic; and the basic function of DWT is rapid attenuation of the periodic function.

(2)Can dual-channel filter banks with perfect reconstruction of the DWT to achieve the original image is decomposed into a plurality of resolution (band), while DCT is not used in the two channel filter banks to realize.

Compared with the DCT, DWT has the following advantages:

(1)High compression rate: in the discrete wavelet transform algorithm, the image can be converted into a series of more effective storage of pixel module "wavelet", therefore, compression ratio based on the DCT transform to increase $10 \% \sim 30 \%$, and the compressed image is more smooth and delicate, quality is better.

(2)Progressive transmission[5]: now the image download DCT compression is to press "block" transmission, therefore only display line by line, and the image DWT compression method to transfer contour data, and then gradually transfer to other data to improve image quality.

(3)Region of interest (Region of Interest, ROI) compression[6 7]: You can specify the area of interest on the picture, and then specify the compression quality during the compression of these areas, or in certain areas of the resumption of the specified compression solution requirements. This is because the wavelet has a locality in space and frequency domains, to fully restore the image of a local, do not need all the coding have been accurately preserved, as long as the corresponding part of it no coding error on it, it is unattainable by existing DCT transform.

\section{Conclusion}

This paper mainly discusses the application of DCT and DWT image compression algorithm based on image in storage field. Based on the DCT compression algorithm has some limitations in the image data analysis and other aspects (such as JPEG compression only less than $64 \mathrm{k} \mathrm{x} 64 \mathrm{k}$ image[8], image quality drop rapidly with the increase of compression ratio, etc.) but the file browse maps without analysis of the application of the image details is quite practical. And compression algorithm based on DWT is able to get a better image quality, JPEG2000 compression speed and reconstruction after the warehouse upon image quality is pretty good, supports lossless compression, and as an international standard, for the future to further improve the warehouse safety system laid the foundation.

\section{Acknowledgement}

In this paper, the research was sponsored by Funding Project for Science and Technology Program of Beijing Municipal Commission of Education 
( KM201310037001 ) 、 Beijing Youth Talent Development Plan (CIT\&TCD201504052)、Beijing Wuzi University Youth Yunhe Scholar、 Beijing Wuzi University Major Program.

\section{References}

1. Xia C.H;Xiong W;Liu Y.Technological information is based Mtatlab DCT transform and its application in image compression [J], 2010, (12):. 249,25.

2. Wang G.W; A discrete cosine transform improved compression algorithm [J] Computer and Information Technology, 2008 (8): 15-18.

3. Wang Y.J; Guo L.Z. Compressed image DCT transform technique [J]. Journal of Henan University Of Urban Construction: Based on 2013.1 (22): 42-46.

4. Sun Y.K;Wavelet transform and images, graphics processing technology [M] Beijing: Tsinghua University Press, 2012.

5. ASKELOF J, Mathias Larsson Carlander Charilaos Christopoulos, Region of interest coding in JPEG2000[J].Signal Processing:Image Communication,(2002)17:105-111.

6. HAMAMOTO K, NISHIMUA T, Basic Investigation on Medical Ultrasonic Echo Image Compression by JPEG200-Availability of Wavelet Transform and ROI Method[A]. 2001 Proceedings of the 23rdAnnual EMBS International Conference[C]. October 25-28,Istanbul:2001.[s.n.],2449-2452.

7. GRGIC S,GRGICM, BRNKAZC, Performance Analysisof Image Compression Using Wavelets, IEEE Transactionson Industrial Electronics,2001,48(3):682-695.

8. Sun S.F; Zhang H.X,;Qiu P.L. JPEG2000- new still image compression standard [J]. Aided Design \& Graphics, 2003,15 (11). 